\title{
Endoperiodontal Lesions and Chicago's New Classification of Periodontal and Peri-implant Diseases and Conditions
}

\author{
Pierre Kuoch ${ }^{1}$, Eric Bonte ${ }^{2}$
}

\begin{abstract}
Aim: To illustrate, with two clinical cases of endoperiodontal lesions, the clinical application of the new classification of periodontal and periimplant diseases and conditions.

Background:The endodont and the periodont are two entities that communicate with each other through physiological communication channels (apical foramen, lateral and secondary canals, and dentinal tubules) resulting in close anatomical and functional interaction. An endoperiodontal lesion is defined by pathological communication between the endodontic and periodontal tissues in a given tooth, according to the definition given by the new classification of periodontal and peri-implant diseases and conditions from the work of the Chicago Consensus Conference in 2017. This new classification differentiates the lesions with and without root damage. Diagnosis and therapeutic strategy will be analyzed through two clinical cases.

Review results: The clinical cases we presented show that the treatment of these lesions must involve endodontic and periodontal management due to the intimate relationship between the tooth and periodontium.

Conclusion: The classification of periodontal and peri-implant diseases and conditions provides a clinical focus on endoperiodontal lesions, based on signs and symptoms that have a direct effect on the prognosis and the treatment of the tooth. The pathological communication between the endodont and the periodontium complicates the management of the involved tooth.

Clinical significance: Chicago's new classification of periodontal and peri-implant diseases and conditions offers an up-to-date vision of periodontal lesions management and highlights the intimate links between endodontic and periodontal tissues.

Keywords: Endodontics, Endoperiodontal lesion, Periodontal diseases.

The Journal of Contemporary Dental Practice (2020): 10.5005/jp-journals-10024-2876
\end{abstract}

\section{BACKGROUND}

The endodont and the periodont are two entities that communicate with each other through physiological communication channels (apical foramen, lateral and secondary canals, and dentinal tubules), resulting in close anatomical and functional interaction. This system evolves without inflammation and remains free from bacterial contamination. The progression of biofilms stops at the epithelial junction and does not concern the endodont.

An endoperiodontal lesion is defined by pathological communication between the endodontic and periodontal tissues of a tooth, ${ }^{1,2}$ according to the definition given by the new classification of periodontal and peri-implant diseases and conditions from the work of the Chicago Consensus Conference in 2017. ${ }^{3}$

This new classification updates the former one proposed in $1999^{4}$ and classifies endoperiodontal lesions and periodontal abscesses as pathologies affecting the periodont. Due to the dual origin of these lesions, their clinical manifestations and their symptomatology will show a multifaceted aspect.

Although relatively rare in clinical practice, endoperiodontal lesions have an adverse impact on tooth prognosis and require a multidisciplinary approach to the diagnosis, treatment, and prognosis of these lesions. ${ }^{5}$ The new classification of endoperiodontal lesions (Table 1) reflects the current clinical state of the lesion, regardless of the cause of the lesion (endodontium or periodontum). The notion of active or chronic lesion is therefore no longer retained. ${ }^{1}$

Two main categories of endoperiodontal lesions are distinguished:

\footnotetext{
1,2Department of Restorative Dentistry and Endodontics, University of Paris, Montrouge, France
}

Corresponding Author: Eric Bonte, Department of Restorative Dentistry and Endodontics, University of Paris, Montrouge, France, Phone: +33682971772, e-mail: eric.bonte@aphp.fr

How to cite this article: Kuoch P, Bonte E. Endoperiodontal Lesions and Chicago's New Classification of Periodontal and Peri-implant Diseases and Conditions. J Contemp Dent Pract 2020;21(7):798-802.

Source of support: Nil

Conflict of interest: None

\section{Endoperiodontal Lesions with Root Damage}

These lesions are associated with traumatic and/or iatrogenic factors: root fracture or cracking, root canal or pulp chamber perforation, or external root resorption.

The prognosis of the tooth is doubtful and its avulsion may be required for these lesions. Indeed, the insertion of a bioactive and watertight material that promotes healing remains quite random and requires either access by opening a flap or an operating microscope. The subsequent coronary restoration will also be weakened by tooth substance loss. Perforations statistically affecting more multirooted teeth, ${ }^{6}$ the management of the furcation area will remain problematic. Root fractures are the main causes of failure following a root perforation filling with the mineral trioxide aggregate (MTA), ${ }^{7}$ even if a healing is observed in the majority of cases, especially if the operator is experienced. ${ }^{8}$

() The Author(s). 2020 Open Access This article is distributed under the terms of the Creative Commons Attribution 4.0 International License (https://creativecommons. org/licenses/by-nc/4.0/), which permits unrestricted use, distribution, and non-commercial reproduction in any medium, provided you give appropriate credit to the original author(s) and the source, provide a link to the Creative Commons license, and indicate if changes were made. The Creative Commons Public Domain Dedication waiver (http://creativecommons.org/publicdomain/zero/1.0/) applies to the data made available in this article, unless otherwise stated. 
Table 1: Classification of endoperiodontal lesions

\begin{tabular}{|c|c|c|}
\hline \multirow{3}{*}{$\begin{array}{l}\text { Endoperiodontal } \\
\text { lesion with root } \\
\text { damage }\end{array}$} & \multicolumn{2}{|c|}{ Root fracture or cracking } \\
\hline & \multicolumn{2}{|c|}{ Root canal or pulp chamber perforation } \\
\hline & \multicolumn{2}{|c|}{ External root resorption } \\
\hline \multirow[t]{6}{*}{$\begin{array}{l}\text { Endoperiodontal } \\
\text { lesion without root } \\
\text { damage }\end{array}$} & \multirow{3}{*}{$\begin{array}{l}\text { Endoperiodontal } \\
\text { lesion in } \\
\text { periodontitis } \\
\text { patients }\end{array}$} & $\begin{array}{l}\text { Grade I: Narrow deep } \\
\text { periodontal pocket in one } \\
\text { tooth surface }\end{array}$ \\
\hline & & $\begin{array}{l}\text { Grade II: Wide deep } \\
\text { periodontal pocket in one } \\
\text { tooth surface }\end{array}$ \\
\hline & & $\begin{array}{l}\text { Grade III: Deep periodontal } \\
\text { pockets in more than one } \\
\text { tooth surface }\end{array}$ \\
\hline & \multirow{3}{*}{$\begin{array}{l}\text { Endoperiodontal } \\
\text { lesion in } \\
\text { nonperiodontitis } \\
\text { patients }\end{array}$} & $\begin{array}{l}\text { Grade I: Narrow deep } \\
\text { periodontal pocket in one } \\
\text { tooth surface }\end{array}$ \\
\hline & & $\begin{array}{l}\text { Grade II: Wide deep } \\
\text { periodontal pocket in one } \\
\text { tooth surface }\end{array}$ \\
\hline & & $\begin{array}{l}\text { Grade III: Deep periodontal } \\
\text { pockets in more than one } \\
\text { tooth surface }\end{array}$ \\
\hline
\end{tabular}

The preexistence of a periapical or periradicular radiolucent image is an unfavorable factor of healing. ${ }^{9}$

\section{Endoperiodontal Lesions without Root Damage}

These lesions are associated with endodontic and periodontal infections. They can be caused by an endodontic infection (case of a carious lesion) that secondarily infects the periodont; a primary periodontal destruction that infects the endodontic secondarily; or a concomitant endodontic and periodontal infection. Lesions with a periodontal primary origin have a lower prognosis than those with an endodontic origin. Endoperiodontal lesions may develop in patients with or without periodontitis. A degraded and, moreover, severe general periodontal terrain greatly reduces the prognosis of the involved tooth. The prognosis of the tooth (favorable, low or nil) may then vary for these lesions, and will also depend on the extension of local periodontal destruction around the tooth: the notion of grade (I-III) is proposed by the classification. Grade III indicates a periodontal destruction extended to more than one tooth surface, weakening the prognosis the most. The possible routes of contamination are therefore multiple, whether from the endodont to the periodont or vice versa.

Endoperiodontal lesions can occur in chronic (fortuitous discovery, often in patients with periodontitis) or acute forms (leading the patient to consult, often related to a traumatic and/or recent iatrogenic event with the presence of an abscess accompanied by pains). The signs and symptoms associated with a tooth affected by these lesions (in patients affected or not by periodontitis) are the presence of a deep periodontal pocket reaching or close to the root apex $(\geq 5 \mathrm{~mm})$ and a negative or altered response to pulp sensitivity tests. ${ }^{2}$ A survey of American endodontists reveals that they prefer to extract a suspected cracked tooth if the depth of the pocket exceeds $6 \mathrm{~mm}^{10}$

Other clinical parameters are reported:

- Bone resorption in the apical or root furcation region

- Spontaneous or percussion and/or palpation pain

- Purulent exudate
- Dental mobility

- One or more sinus tracks

- An alteration in the color of the gum and/or clinical crown.

The shape of the periodontal pocket, given by probing, may differentiate a sinus tract of the endodontic origin with a purely periodontal origin defect or a combined endoperiodontal lesion. However, this test remains quite tricky from a clinical point of view. ${ }^{11,12}$ With the new classification, the shape of the periodontal pocket is no longer relevant for the diagnosis of the endoperiodontal lesion since a deep probing combined with a negative or altered sensitivity test is sufficient to diagnose it, regardless of the coronary flare of the probing. However, its usefulness is relevant in determining prognosis. Indeed, the more extensive the probing is on the tooth surface, the weaker the prognosis becomes.

The diagnostic approach to endoperiodontal lesions will therefore be carried out by this new classification: an examination of the current status of the lesion, the prognosis of the affected tooth, and the maintenance and treatment of the tooth or its avulsion.

- First step: After reporting the presence of a deep periodontal pocket, reaching or approaching the root apex, associated with a negative or altered response to pulp sensitivity tests, the first step is to detect the presence or not of a loss of the root surface integrity. It is important to collect the patient's oral history in order to identify any trauma or any endodontic and coronoradicular restoration treatments that may be the cause of iatrogenic injury. If a traumatic and/or iatrogenic event is identified, then a clinical and radiographic examination is performed to reveal the presence of an endodontic perforation, a fracture, or root resorption. Such a diagnosis has a nil or low prognosis, and the treatment is often avulsion or root amputation of the tooth.Particular attention should be paid to the existence of palatal dental invagination with root extension, which can mimic a vertical root fracture on radiography when a periodontal lesion is located at this anatomical feature.

- Second step: Once root damage has been ruled out, a thorough examination of the current periodontal status must be performed. The patient will then be placed in a periodontal disease context or not. The prognosis is determined on whether or not periodontitis is present and its severity, as well as the extent of local periodontal destruction around the tooth (grades I-III). If it appears that the tooth can be maintained on the arch, the management required for these lesions is both endodontic and periodontal.

The treatment sequence always begins with a chemomechanical cleaning of the endodontium and its obturation when the conditions for filling are met (dry canal and in the absence of symptoms). In the absence of periodontal disease, endodontic treatment alone may be sufficient, especially when the periodontal lesion is limited. If healing is not observed, the search for an infectious gateway of periodontal origin or related to root damage must be thoroughly explored. When the lesion has a combined periodontal origin, nonsurgical periodontal debridement can be initiated to promote the healing of the endoperiodontal lesion. ${ }^{13,14}$ An immediate periodontal surgical treatment has not demonstrated superiority over a 3-month delay. ${ }^{15}$ In case of doubt about a periodontal origin, it therefore seems prudent to defer the decision to intervene on the periodontium. When the periodontal component is important (particularly in cases of periodontal abscess) and the obturation conditions are not met, an intracanal medication based on calcium hydroxide is put in place associated with a temporary watertight 
coronary restoration, leaving time for periodontal debridement. In most cases, nonsurgical periodontal debridement combined with oral hygiene instructions will be sufficient in this first phase of periodontal therapy. ${ }^{13}$ After disappearance or a significant improvement of periodontal problems, endodontic obturation can therefore be initiated, followed by a coronary restoration. Continued periodontal treatment is essential to ensure the best possible healing of these lesions. Endodontic microsurgery may be a therapeutic alternative. However, in a retrospective study of 414 cases, Song et al. ${ }^{16}$ showed that this surgery performed in a context of the endoperiodontal lesion had a poorer prognosis at 12 years than when the etiology was purely endodontic.

\section{Case Descriptions}

\section{Case 1}

A 40-year-old male patient, with no medical-surgical history, came to the department of oral medicine at the Bretonneau Hospital (Assistance Publique-Hôpitaux de Paris) for periodontal assessment. Clinical examination revealed the presence of two sinus tracks facing the first left mandibular molar (\#36): one on the vestibular mucosa next to the mesial root and the other opening into the periodontal pocket facing the distal root (Fig. 1). Examination of the dental arch in the area showed that there was no decayed tooth and noncarious lesion. The examination revealed the absence of an iatrogenic and/or traumatic event associated with this tooth. Periodontal examination diagnosed a stage III/ grade III periodontitis. ${ }^{17}$ The pulp sensitivity test (Pulpofluorane, Septodont) of the tooth \#36 was negative and the periodontal probing gave a wide and deep pocket on the distobuccal surface of

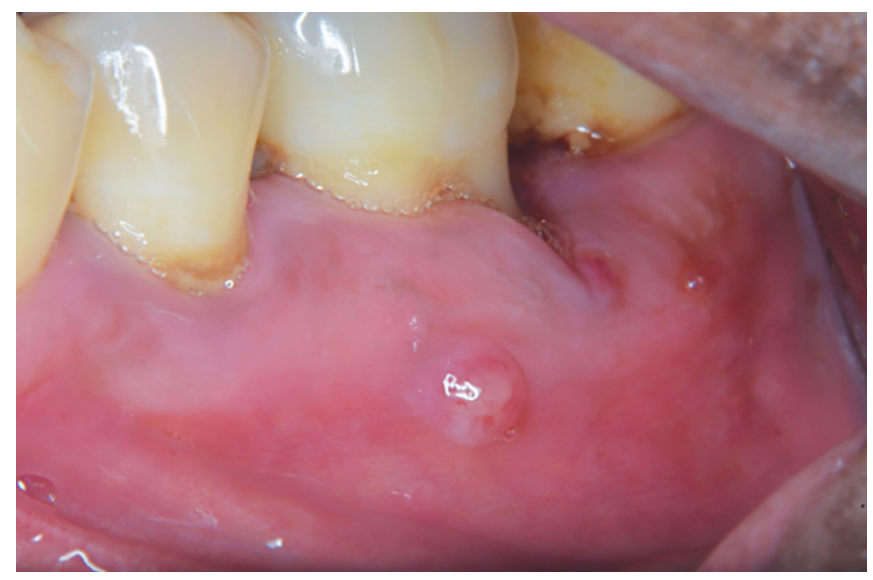

Fig. 1: Clinical view of sinus tracks facing the tooth \#36

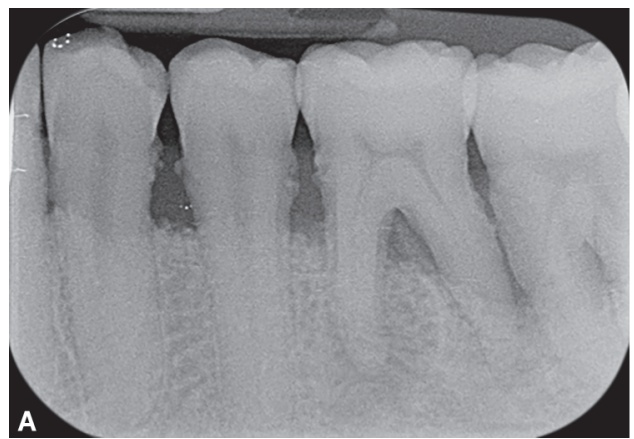

Figs 2A and B: (A) Retroalveolar radiograph centered on tooth \#36; (B) Eccentric radiograph: PAI 5 on both roots
Fig. 3: Clinical view of the vestibular mucosa facing the tooth \#36 at 2 weeks after the first endodontic disinfection

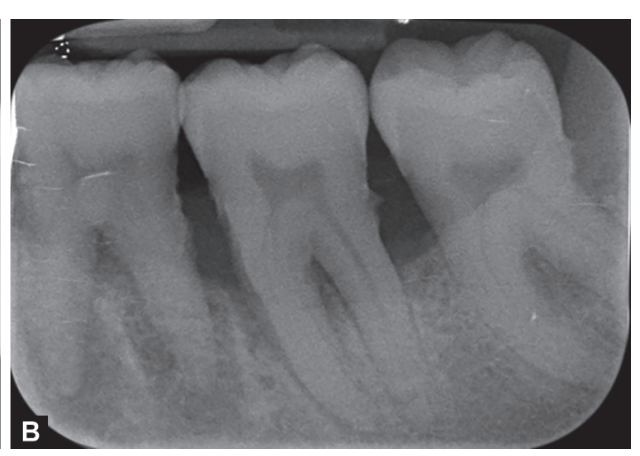

the tooth. Retroalveolar radiograph (VistaScan Mini Easy, DBSWIN, Dürr Dental) showed severe alveolysis in the distal root of the tooth \#36 with interradicular alveolysis, giving a degree II $^{18}$ in the periodontal destruction in the furcation area, measured with a Nabers probe. A periradicular inflammatory lesion of endodontic origin was objectified at the mesial and distal roots (periapical score index PAI $5^{19}$ ) (Fig. 2). The diagnosis established for the tooth \#36 was an endoperiodontal lesion without root damage of grade II, in the periodontitis patient.

The therapeutic decision was to maintain the tooth on the arch and to add an endodontic and periodontal treatment: a first chemomechanical disinfection of the endodontium was carried out under rubber dam (Hygienic Dental Dam, Coltene), by root canal cleaning with sodium hypochlorite $3 \%$ solution (Parcan, Septodont) and shaping (Protaper Gold, Dentsply), followed by an intracanal calcium hydroxide-based preparation filling and a provisional restauration with a glass-ionomer cement (Fuji IX, GC). After a delay of 2 weeks, the sinus tracks had disappeared (Fig. 3) and the obturation of the endodontium was undertaken: final disinfection with sodium hypochlorite $3 \%$ solution (Parcan, Septodont) and EDTA 17\% solution (EDTA17\%, Produits Dentaires SA), finished by sodium hypochlorite $3 \%$ solution, drying with sterile paper points (Paper Points, VDW), and obturation following a warm vertical compaction technique (Elements Free Downpack, SyndronEndo; Root canal Plugger, Dentsply-Maillefer) of gutta-percha (Hygienic Gutta Percha Points, Coltene) impregnated with zinc oxide eugenol cement (Pulp Canal Sealer, Kerr). The coronal space of the canal was obturated with warm gutta-percha by the MacSpadden rotative technique (Gutta Condensor Size 25, Dentsply) (Fig. 4A). Periodontal

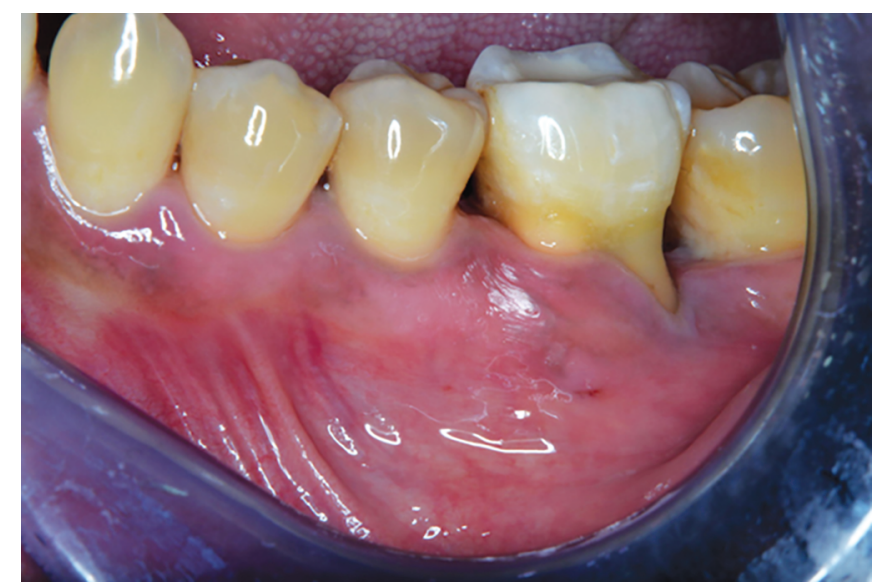



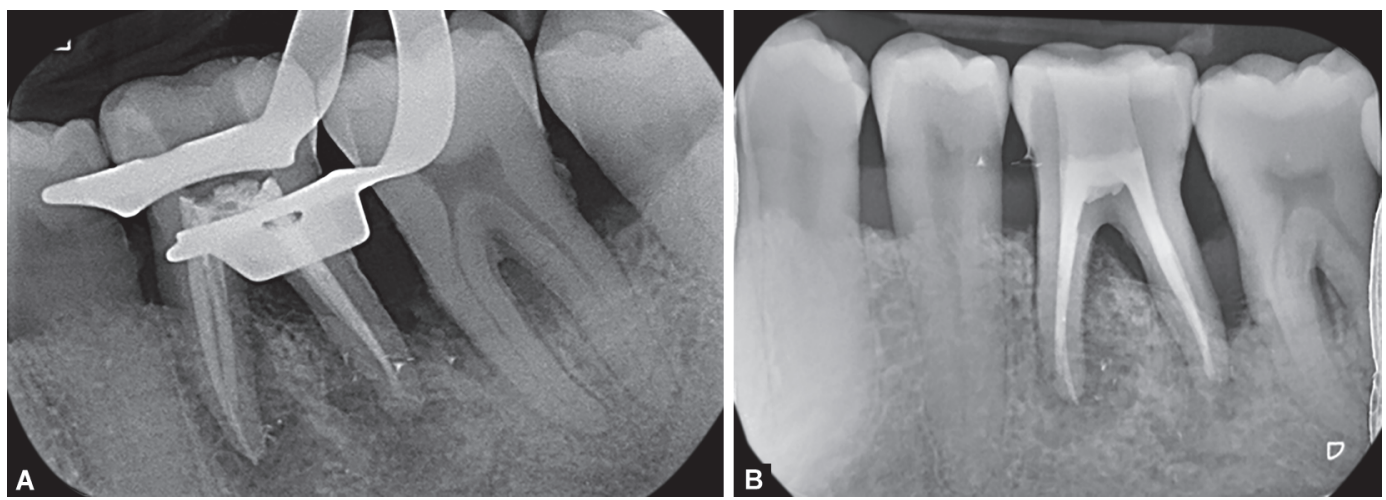

Figs 4A and B: (A) Postoperative retroalveolar radiograph; (B) 6 months' follow-up
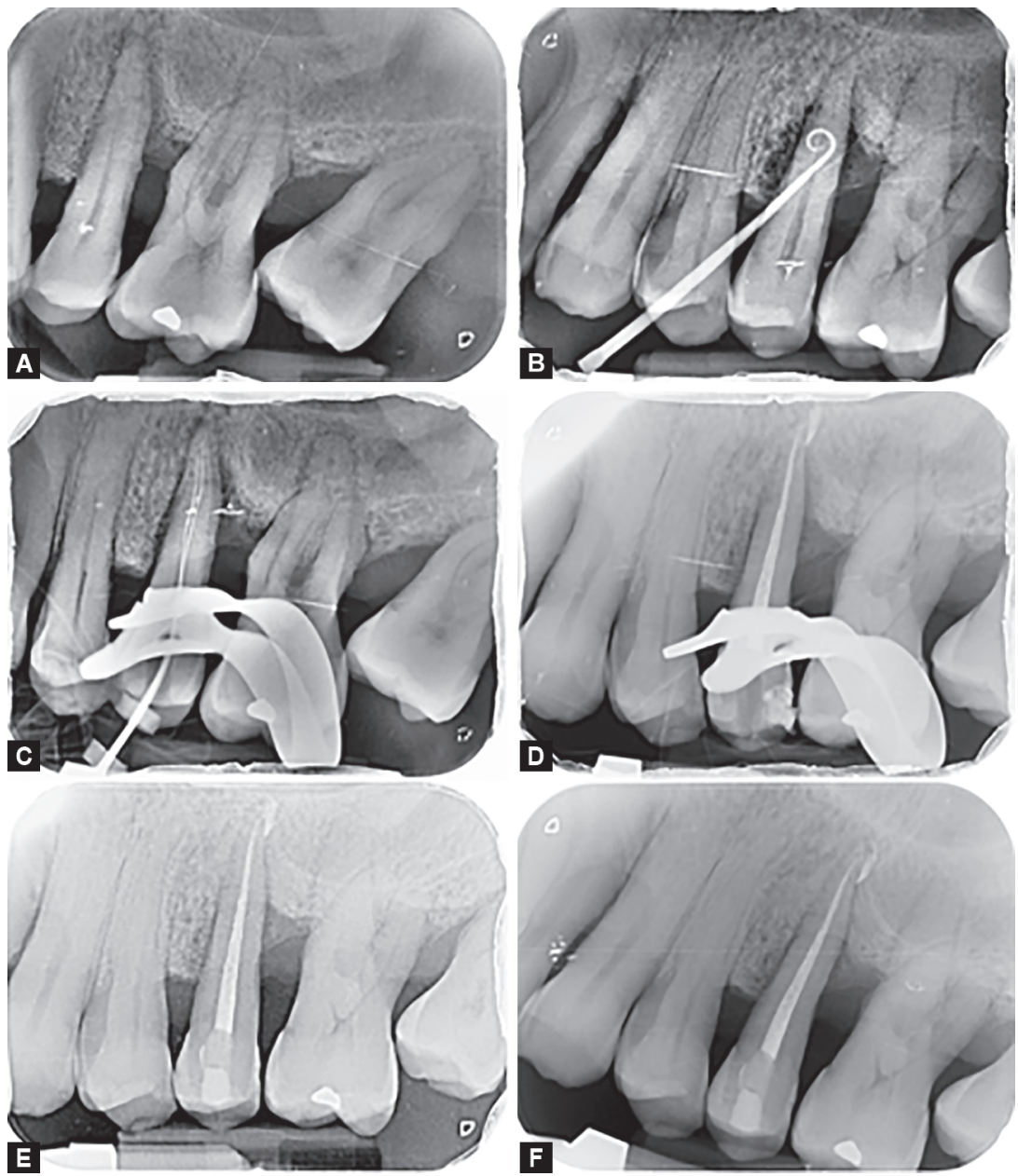

Figs 5A to F: (A) Retroalveolar radiograph centered on the tooth \#25; (B) Radiograph with gutta-percha cone into sinus track; (C) Preoperative radiograph: two separate canals; (D) Postoperative radiograph; (E) 6 months' follow-up; (F) 1.5 years' follow-up

debridement was therefore initiated and the tooth remained symptom-free at 6 months of follow-up (Fig. 4B).

\section{Case 2}

A 60-year-old male patient, in good general health, was followed in the oral medicine department of the Bretonneau Hospital (Assistance Publique-Hôpitaux de Paris) for a stage III/grade I periodontitis. ${ }^{17}$

Clinical examination revealed the absence of cavities and noncarious lesions, but attested to the presence of a sinus track on the vestibular mucosa of the second upper-left premolar (tooth \#25). The pulp sensitivity test (Pulpofluorane, Septodont) was negative and the periodontal probing was wide and deep on the distovestibular surface of the tooth. A retroalveolar radiograph (VistaScan Mini Easy, DBSWIN, Dürr Dental) (Fig. 5A) was then performed with the introduction of a fine gutta-percha cone (Hygienic Gutta Percha Points, Coltene) through the sinus track (Fig. 5B). It showed an enlargement of the periodontal ligament and a severe angular alveolysis in distal of the tooth \#25. The path 


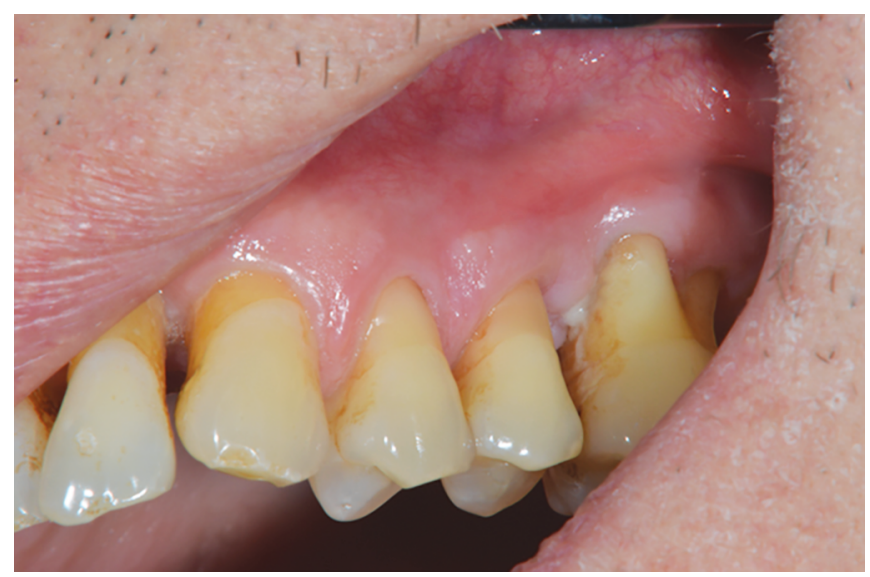

Fig. 6: Clinical view of the vestibular mucosa at 1.5 years: absence of sinus track

of the gutta-percha cone had suggested a periodontal lesion origin of the sinus track but may had an endodontic origin related to a lateral canal. The diagnosis established for the tooth \#25 was an endoperiodontal lesion without root damage of grade II, in the periodontitis patient.

The therapeutic decision was to keep the tooth on the arch. The combined endoperiodontal treatment would first consist of an endodontic cleaning with sodium hypochlorite $3 \%$ solution (Parcan, Septodont) and shaping (Reciproc, VDW) under rubber dam (Hygienic Dental Dam, Coltene) with an intersession of calcium hydroxide dressing and provisional coronal restauration (Fuji IX, GC). Because of the persistence of sinus track at the end of this 2-week session (confirming a periodontal sinus track origin), a nonsurgical periodontal debridement was performed in the meantime and the sinus track disappeared. The endodontic obturation could be then undertaken following the same protocol and technique as described in the first clinical case (Figs $5 \mathrm{C}$ to $\mathrm{E}$ ). The 1.5 year prognosis was favorable (Figs $5 \mathrm{~F}$ and 6).

\section{Conclusion}

The new classification of periodontal and peri-implant diseases and conditions provides a clinical focus on endoperiodontal lesions. It is based on signs and symptoms that have a direct effect on the prognosis and on the treatment of the tooth, such as the presence or the absence of root fractures or perforations, the presence or the absence of periodontitis, and the extent of periodontal destruction around the affected tooth. The clinical cases we presented show that the treatment of these lesions must involve endodontic and periodontal care due to the intimate relationship between the tooth and the periodontium.

\section{Clinical Significance}

Chicago's new classification of periodontal and peri-implant diseases and conditions offers an up-to-date vision of periodontal lesions management and highlights the intimate links between endodontic and periodontal tissues.

\section{References}

1. Papapanou PN, Sanz M, Buduneli N, et al. Periodontitis: consensus report of workgroup 2 of the 2017 world workshop on the classification of periodontal and peri-implant diseases and conditions: classification and case definitions for periodontitis. J Periodontol 2018;89(Suppl 1):S173-S182.

2. Herrera D, Retamal-Valdes B, Alonso B, et al. Acute periodontal lesions (periodontal abscesses and necrotizing periodontal diseases) and endo-periodontal lesions. J Clin Periodontol 2018;45(Suppl 20):S78-S94.

3. Caton JG, Armitage G, Berglundh T, et al. A new classification scheme for periodontal and peri-implant diseases and conditionsintroduction and key changes from the 1999 classification. J Clin Periodontol 2018;45(Suppl 20):S1-S8.

4. Armitage GC. Development of a classification system for periodontal diseases and conditions. Ann Periodontol 1999;4(1):1-6. DOI: 10.1902/ annals.1999.4.1.1.

5. Tsesis I, Nemcovsky CE, Nissan J, et al. Endodontic-Periodontal Lesions: Evidence-Based Multidisciplinary Clinical Management. Springer; 2019.

6. Tsesis I, Rosenberg E, Faivishevsky V, et al. Prevalence and associated periodontal status of teeth with root perforation: a retrospective study of 2,002 patients' medical records. J Endod 2010;36(5):797-800. DOI: 10.1016/j.joen.2010.02.012.

7. Mente J, Hage N, Pfefferle T, et al. Treatment outcome of mineral trioxide aggregate: repair of root perforations. J Endod 2010;36(2):208-213. DOI: 10.1016/j.joen.2009.10.012.

8. Mente J, Leo M, Panagidis $D$, et al. Treatment outcome of mineral trioxide aggregate: repair of root perforations-long-term results. J Endod 2014;40(6):790-796. DOI: 10.1016/j.joen.2014.02.003.

9. Siew K, Lee AHC, Cheung GSP. Treatment outcome of repaired root perforation: a systematic review and meta-analysis. J Endod 2015;41(11):1795-1804. DOI: 10.1016/j.joen.2015.07.007.

10. Abulhamael AM, Tandon R, Alzamzami ZT, et al. Treatment decisionmaking of cracked teeth: survey of american endodontists. J Contemp Dent Pr 20(5):543-547.

11. Al Shayeb KNA, Turner W, Gillam DG. Periodontal probing: a review. Prim Dent J 2014;3(3):25-29. DOI: 10.1308/205016814812736619.

12. Listgarten MA. Periodontal probing: what does it mean? J Clin Periodontol 1980;7(3):165-176. DOI: 10.1111/j.1600-051X.1980. tb01960.x.

13. Abbott $P$, Salgado JC. Strategies for the endodontic management of concurrent endodontic and periodontal diseases. Aust Dent J 2009;54(Suppl 1):S70-S85.

14. Gupta S, Tewari S, Tewari S, et al. Effect of time lapse between endodontic and periodontal therapies on the healing of concurrent endodontic-periodontal lesions without communication: a prospective randomized clinical trial. J Endod 2015;41(6):785-790. DOI: 10.1016/j.joen.2015.02.015.

15. Tewari S, Sharma G, Tewari S, et al. Effect of immediate periodontal surgical treatment on periodontal healing in combined endodonticperiodontal lesions with communication-a randomized clinical trial. J Oral Biol Craniofacial Res 2018;8(2):105-112. DOI: 10.1016/j. jobcr.2018.04.002.

16. Song M, Kang M, Kang DR, et al. Comparison of the effect of endodontic-periodontal combined lesion on the outcome of endodontic microsurgery with that of isolated endodontic lesion: survival analysis using propensity score analysis. Clin Oral Investig 2018;22(4):1717-1724. DOI: 10.1007/s00784-017-2265-1.

17. Chapple ILC, Mealey BL, Van Dyke TE, et al. Periodontal health and gingival diseases and conditions on an intact and a reduced periodontium: consensus report of workgroup 1 of the 2017 world workshop on the classification of periodontal and peri-implant diseases and conditions. J Periodontol 2018;89(Suppl 1):S74-S84.

18. Hamp S-E, Nyman S, Lindhe J. Periodontal treatment of multi rooted teeth. J Clin Periodontol 1975;2(3):126-135. DOI: 10.1111/j.1600051X.1975.tb01734.x.

19. Ørstavik D, Kerekes K, Eriksen HM. The periapical index: a scoring system for radiographic assessment of apical periodontitis. Dent Traumatol 1986;2(1):20-34. DOI: 10.1111/j.1600-9657.1986. tb00119.x. 